\title{
Angular dependence of optical second-harmonic generation from a monolayer grating
}

\author{
R.W.J. Hollering, Q.H.F. Vrehen \\ Philips Research Laboratories, P.O. Box 80.000, 5600 JA Eindhoven, The Netherlands
}

and

G. Marowsky

Max-Planck-Institut für Biophysikalische Chemie, Postfach 2841, 3400 Göttingen, Fed. Rep. Germany

Received 23 March 1990; revised manuscript received 14 May 1990

\begin{abstract}
Experimental results on the variation of the SH signal with angle of incidence for different diffraction orders of a monolayer grating are presented. For specular reflection the data are in good agreement with recently published theory. The angular dependence of the power in the non-zero grating orders is well described by a slight extension of this theory. The spatial profile structured in the monolayer is determined from power ratios of the diffracted orders. The results are in good agreement with predictions based on a model for thermal desorption, with a surface temperature calculated from a heat conduction equation.
\end{abstract}

\section{Introduction}

Optical second-harmonic generation ( $\mathrm{SHG}$ ) has proven to be an effective and sensitive technique for studying surface and interface properties. The surface-specific character of the technique arises from symmetry considerations, which in the electric dipole limit prohibit second-order nonlinear optical processes in the bulk of centrosymmetric media. At interfaces or surfaces where the inversion symmetry is broken, the dipolar SHG process is allowed $[1,2]$. The technique has been extensively used to study various properties of adsorbed molecular monolayers, such as molecular orientation, hyperpolarizability, adsorption and desorption kinetics, phase transitions, molecule-molecule and molecule-substrate interactions [1-3].

An interesting extension of the above-mentioned studies deals with periodic modulations in adsorbate density. Recently Zhu et al. [4,5] created a monolayer grating by thermal desorption of an adsorbate by exposing it to intense interfering laser beams. Subsequently, changes in the diffracted intensity were measured due to smearing out of the grating caused by surface diffusion of the adsorbed molecules. Reider et al. [6] exploited the fact that on a monolayer grating the fundamental beam is not diffracted, which implies that the diffracted SH intensity can be measured background-free. They showed that a grating structure allows the study of adsorbates on top of noncentrosymmetric substrates, where it is normally hard to detect the weak surface SH signal against the background signal of the bulk. Suzuki and Heinz [7] determined the density profile of a monolayer grating from the intensities of the diffracted grating orders. They compared the obtained grating profile with predictions of a model based on thermal desorption. Bratz et al. [8] extensively discussed the use of monolayer gratings on top of linear optical planar waveguides with respect to second-harmonic generation with high conversion efficiency. In this case the grating wavevector, related to the periodic modulation in the adsorbate, is used to achieve phasematching of fundamental and second-harmonic lightwaves.

For a quantitative interpretation of data from gratings at surfaces, analogous to previous studies on gratings in bulk material [9], the optical response of 
a surface nonlinear grating should be accurately known. In the earlier work referred to above, the grating constant was always large compared to the wavelength. As a result, the various grating orders showed only a small angular dispersion, and consequently the angular dependence did not differ greatly from that for specular diffraction. In the present paper experimental data are reported for a grating with a period of $1.6 \mu \mathrm{m}$, comparable to the wavelength of the fundamental wave $(1.06 \mu \mathrm{m})$. The observed variation of the second-harmonic $(\mathrm{SH})$ power with incident (or diffracted) angle is presented for several grating orders. A slight modification of the theory for specular reflection leads to good agreement with the data. It is thus possible to deduce the spatial profile structured in the monolayer from the observed intensity ratios. The profile can be reasonably explained on the basis of a photothermal process with which the grating is written in the layer.

\section{Experimental}

The experimental set-up is shown in fig. 1. The measurements were performed with an active-passive modelocked Nd-YAG laser which emits pulses of $30 \mathrm{ps}$ duration at a wavelength of $1.064 \mu \mathrm{m}$ and a repetition rate of $10 \mathrm{~Hz}$. The laser beam, which is transmitted through a long-pass filter, a polarizer and a half-wave plate, is directed unfocused onto the sample surface (beam diameter $2.5 \mathrm{~mm}$ ). Typical energies in the experiments were $1 \mathrm{~mJ} /$ pulse. To reduce the effect of pulse-to-pulse intensity fluctuations the signal is normalized to a SH signal generated in quartz. The sample and the complete detection system, consisting of filters, analyser, monochromator and photomultiplier are mounted on a $\theta-2 \theta$ goniometer. The system allows measurements for incident angles ranging from $20^{\circ}$ to $90^{\circ}$ with respect to the surface normal. The goniometer and all polarizing components are stepping-motorcontrolled. The sample stage can be decoupled from the detector arm to allow for independent setting of angle of incidence and angle of observation. It should be noted that the average second-harmonic power is measured and not the average second-harmonic intensity, because the detector area is large compared to the cross-section of the beam.

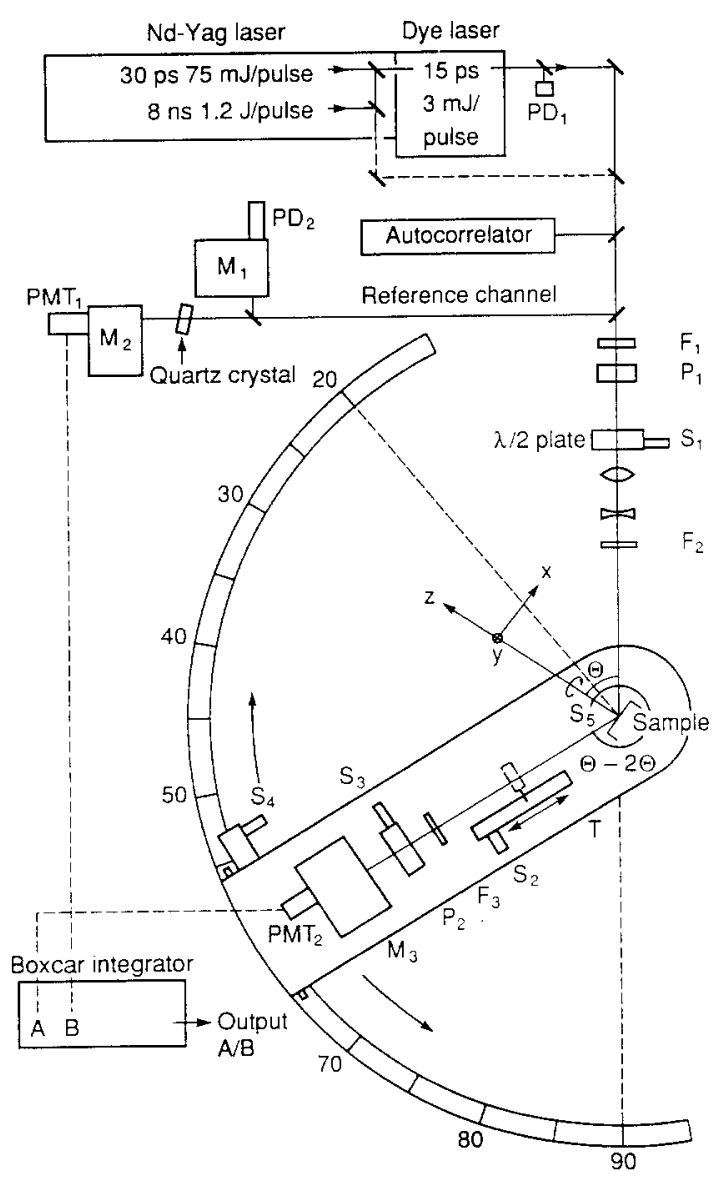

Fig. 1. The experimental set-up for second-harmonic generation at surfaces consisting of a double-cavity nano-pico-second NdYAG laser, a picosecond dye laser, and an autocorrelator. For angular dependence studies the complete detection system is mounted on a $\theta-2 \theta$ goniometer. For phase measurements of the $\mathrm{SH}$ light a reference sample is mounted on a translation stage $T$. The other symbols denote: polarizers $P_{1,2}$, filters $F_{1,2,3}$, monochromators $\mathrm{M}_{1,2,3}$, photomultipliers $\mathrm{PMT}_{1,2}$, photodiodes $\mathrm{PD}_{1,2}$, stepping motors $S_{1-5}$. The sample stage can be decoupled from the detector arm, so that the angles of incidence and of observation can be adjusted independently.

Monolayers were deposited on glass substrates by spincoating a solution of hemicyanine [10] in isopropanol at $200 \mathrm{rev} / \mathrm{min}$. The writing of the small period gratings in these monolayers was made possible by the availability of facilities for high-density optical recording in the optics group of our laboratory. Partial desorption of the monolayer was per- 
formed by focusing a $50 \mathrm{~mW}$ Ar-ion laser operating at $\lambda=457.9 \mathrm{~nm}$ to a spot of $0.7 \mu \mathrm{m}$. With an effective irradiation time of $140 \mathrm{~ns}$ the power density of 13 $\mathrm{MW} / \mathrm{cm}^{2}$ in the absorption region of the hemicyanine was sufficient to ablate the monolayer partially. With a mechanical reproducibility of $20 \mathrm{~nm}$ high-quality gratings of several square centimeters in area containing 610 lines $/ \mathrm{mm}$ were obtained.

\section{Theory}

The second-harmonic intensity is usually calculated for the case that the angle of incidence equals the angle of reflection, see e.g. refs. [11] and [12]. For reflection in higher orders off a grating these angles may be quite different and a modification of the theory is required. Following the arguments presented by Mizrahi and Sipe [12], we derive for the second-harmonic power $P_{2 \omega}^{(n)}\left(\theta_{n}\right)$ in the $n$th grating order as a function of diffracted angle $\Theta_{n}$ :

$$
\begin{aligned}
& P_{2 \omega}^{(n)}\left(\Theta_{n}\right) \propto \sec \Theta_{0} \sec \Theta_{n} \\
& \quad \times\left|\boldsymbol{e}_{2 \omega} \cdot \chi_{n}^{(2)}: \boldsymbol{e}_{\omega} \cdot \boldsymbol{e}_{\omega}\right|^{2} P_{\omega}^{2}\left(\Theta_{0}\right) \mid A,
\end{aligned}
$$

$P_{\omega}\left(\Theta_{0}\right)$ is the power of the fundamental beam with cross-section $A$ and incident angle $\boldsymbol{\theta}_{0}$. For an incident wavelength $\lambda$, the relationship between $\Theta_{n}$ and $\Theta_{0}$ for a grating with period $d$ is given by $\sin \Theta_{n}=$ $\sin \Theta_{0}+n \lambda / 2 d$. For specular reflection, $\Theta_{n}=\Theta_{0}$, eq. (1) reduces to eq. (5.1) of ref. [12]. The vectors $e_{\omega}$ and $\boldsymbol{e}_{2 \omega}$ are defined in table 2 of ref. [12]. For the calculation of $e_{\omega}$ the Fresnel factors must be taken at the incident angle $\Theta_{0}$, whereas for the calculation of $\boldsymbol{e}_{2 \omega}$ they must be taken at the diffracted angle $\boldsymbol{\Theta}_{n}$. The intensity of the harmonic beam is proportional to $\sec ^{2} \theta_{n}$, whereas the cross-sectional area is proportional to $\sec \boldsymbol{\theta}_{0} \cos \Theta_{n}$, so that the total power is proportional to $\sec \theta_{0} \sec \theta_{n}$.

For an adsorbate in which the molecules have no preferred direction in the substrate plane the secondorder nonlinear susceptibility tensor $\chi^{(2)}$ contains the components $\chi_{z z z}^{(2)}, \chi_{z i i}^{(2)}$ and $\chi_{i z i}^{(2)}$, where $i=x, y$ are parallel to the surface and $z$ coincides with the surface normal (see fig. 1). By choosing s-polarized excitation and p-polarized detection only the element $\chi_{z y y}^{(2)}$ is involved $[1,2]$. This tensor element just acts as a scaling factor, and does not influence the an- gular dependence of the signal, which is now completely determined by a combination of Fresnel factors of fundamental and second-harmonic light waves, and the geometrical factors in eq. (1).

The intensity of the $n$th grating order is related to the $n$th Fourier component $\chi_{n}^{(2)}$ of the spatial modulation in $\chi^{(2)}(x)$, which is given by

$\chi^{(2)}(x)=\chi^{(2)}+2 \sum_{n=1} \chi_{n}^{(2)} \cos n \pi x / d$.

Here $\chi \delta^{2)}$ is the average susceptibility. At first it was assumed by us that the writing process described in section 2 would lead to a $100 \%$ modulation in adsorbate density and thus in $\chi^{(2)}$. The experimental data, however, show that the modulation depth is much smaller than $100 \%$.

To obtain an estimate of the modulation to be expected, the thermal desorption of a hemicyanine monolayer is studied. With the rate of desorption $\mathrm{d} N / \mathrm{d} t=-\nu N \exp \left(-E_{\mathrm{des}} / k_{\mathrm{B}} T\right)[13]$ where $k_{\mathrm{B}}$ is the Boltzman constant, the surface coverage $N(t)$ normalized against that of a full monolayer depends on the thermal history $T(t)$, and is given by

$N(t)=\exp \left(\int_{T_{0}}^{T(t)} \frac{-\nu}{\mathrm{d} T^{\prime} / \mathrm{d} t} \exp \left(-E_{\mathrm{des}} / k T^{\prime}\right) \mathrm{d} T^{\prime}\right)$.

For a known change of sample temperature $\left(\mathrm{d} T^{\prime} / \mathrm{d} t\right)$ with time, and a given value of the preexponential factor $\nu$, the desorption energy $E_{\text {des }}$ can be determined.

The surface temperature, during the writing of the monolayer grating, follows from the heat conduction equation

$\rho c \partial T(\boldsymbol{r}, t) / \partial t-K \nabla^{2} T(\boldsymbol{r}, t)=S(\boldsymbol{r}, t)$.

Here the substrate density $\rho$, specific heat $c$ and thermal conductivity $K$ are taken to be temperature-independent. $S(\boldsymbol{r}, t)$ is the energy source term, located at the surface $\left(z=z_{0}\right)$, which is given by

$S(\boldsymbol{r}, t)=I_{\text {abs }}(N) g(t) f(r) \delta\left(z-z_{0}\right)$.

Here $I_{\text {abs }}(N)$ is the absorbed light intensity, and $f(r)$ and $g(t)$ are the normalized radial and temporal profile of the laser beam. The surface temperature $T(r, t)$ for illumination by means of a laser with a gaussian beam profile $\left(f(r)=\exp (-r / d)^{2}\right)$ and a gaussian time profile $\left(g(t)=\exp (-t / \rho)^{2}\right)$ has been 
given by Bechtel [14]. In the simplified situation of a uniform illumination with constant intensity $(f(r)=$ constant, $g(t)=$ constant $)$ and a heat flow only in a direction normal to the surface, the surface temperature is given as

$$
T(t)=T_{0}+2 I_{\text {abs }}(N) \sqrt{t / \pi K \rho c} .
$$

Here $T_{0}$ is the initial substrate temperature.

\section{Results}

We first consider the specularly reflected light ( $n=0$ curve in fig. 2 ). The variation of the $\mathrm{SH}$ signal with angle of incidence is completely determined by the Fresnel coefficients, which depend on the accurately known substrate refractive indices 1.5067 and 1.5188 at $\lambda=1064 \mathrm{~nm}$ and $\lambda / 2=532 \mathrm{~nm}$. Excellent agreement with theory is obtained without any ad-

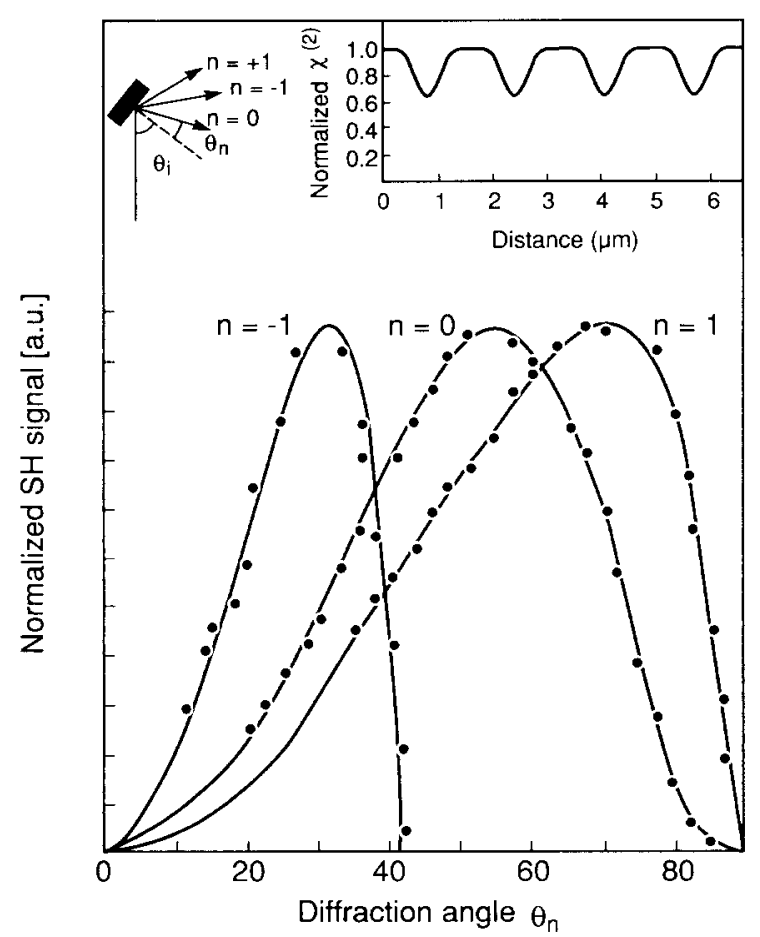

Fig. 2. Measured and calculated variation of the second-harmonic power as a function of diffracted angle $\theta_{n}$ for the orders $n=-1, n=0$ and $n=1$ of a monolayer grating. The inset shows the calculated spatial modulation of $\chi^{(2)}$ created by partial desorption of the adsorbed monolayer. justable parameters. Equally good agreement with theory was also reported by Guyot-Sionnest et al. [15] for the total reflection geometry. They also pointed out that an earlier transmission experiment [16] showed discrepancies with theory. Recent work [17] indicates that multiple reflections in the substrate may have affected the experimental data.

Next we consider diffraction. Both in transmission and reflection grating orders from $n=-3$ to $n=3$ could be detected. From the relationship between incident and diffracted angles we determined the grating period $d$ to be $1.64 \mu \mathrm{m}$. The measured and calculated variation of the SH power as a function of the diffracted angle for the reflected orders $n=-1$ and $n=1$ is shown in fig. 2. Good agreement is found. We may thus rely on the data to determine the Fourier coefficients $\chi_{n}^{(2)}$ from the relative intensities of the diffracted orders. For a $100 \%$ square-wave-like modulation $\chi_{n}^{(2)}$ is given by $\chi_{n}^{(2)}=$ $\left(\chi^{(2)} / n \pi\right) \sin (n \pi a / d)$, where $a / d$ is the fraction of covered substrate. As follows from this expression, the diffracted intensities are zero for $a / d=0$ (no monolayer on the substrate) and $a / d=1$ (full monolayer), while for $a / d=0.5$ the even order of the grating vanish. For a square-wave modulation with depth $M$ the Fourier components of the higher orders scale with a factor $M$, whereas the zeroth order scales with $(1-M)+M a / d$. To measure the relative intensities of the diffracted orders the detector was set at a fixed angle of $95^{\circ}$ with respect to the incident beam. The grating was then rotated to direct the diffracted beam onto the detector. For $n=2, n=1, n=0$ and $n=-1$ the power ratios $1: 8.3: 375: 1.2$ were observed. The calculated powers for $a / d=0.6$ and a $20.8 \%$ squarewave-like modulation in $\chi^{(2)}$ are $1: 8.8: 375: 1.7$. The small difference may be further reduced by using a gaussian spatial modulation. The result of $a / d=0.60$ is in agreement with the ratio of 0.57 following from the $0.7 \mu \mathrm{m}$ focus spotsize to the $1.64 \mu \mathrm{m}$ grating period. The modulation in $\chi^{(2)}$ obtained with use of eq. (2) and the measured power ratios is sketched in the inset of fig. 2.

The modulation depth, created in the monolayer, depends on the desorption energy of hemicyanine on glass and the surface temperature. The desorption energy can be estimated from the data in fig. 3 . Here the variation in the SH signal is shown during thermal desorption of the monolayer, for a linear tem- 


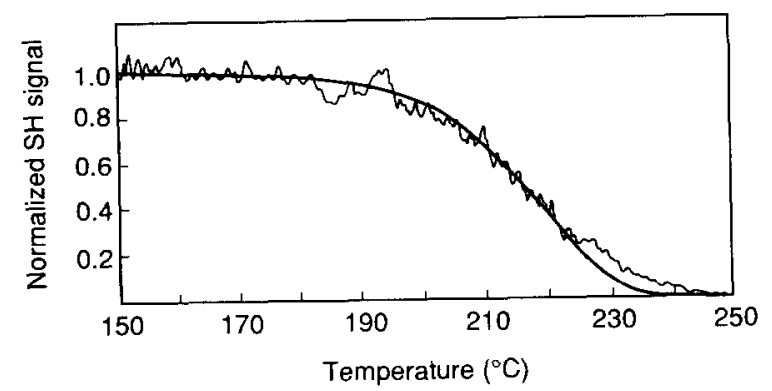

Fig. 3. Measured and calculated variation of the second-harmonic signal during the thermal desorption of a hemicyanine monolayer on glass.

perature ramp of $8^{\circ} \mathrm{C} / \mathrm{min}$. The data are well described by eq. (3), which is valid for a first-order desorption process [13]. A unique determination of both $\nu$ and $E_{\text {des }}$ is not possible from experiments with just one single heating rate. The experimental desorption curve can be fitted with use of eq. (3) for a range of $\nu$ values and corresponding values of $E_{\mathrm{des}}$. A frequency $\nu=10^{13} \mathrm{~s}^{-1}$ is commonly used in the literature [13]. For $\nu$ ranging from $10^{12} \mathrm{~s}^{-1}$ to $10^{14} \mathrm{~s}^{-1}$, we obtain that $E_{\text {des }}$ varies from $1.38 \mathrm{eV}$ to $1.56 \mathrm{eV}$. These values for $E_{\text {des }}$ are comparable to those obtained by Mullin et al. [18] for desorption of liquid crystal monolayers on glass. The temporal evolution of the surface temperature calculated with use of eq. (6) for a monolayer absorption of $2 \%$ at $\lambda=457.9$ $\mathrm{nm}$, a density $\rho=2.6 \times 10^{3} \mathrm{~kg} / \mathrm{m}^{3}$, a thermal conductivity $K=1 \mathrm{~W} / \mathrm{m} \mathrm{K}$ and specific heat $c=0.84 \times$ $10^{3} \mathrm{~J} / \mathrm{kg} \mathrm{K}$ for glass, is $T(t)=T_{0}+2.0 \times 10^{6} \sqrt{t}$. Using these numbers we calculated for an irradiation time of $140 \mathrm{~ns}$ a modulation depth between $3 \%$ and $30 \%$ for $\nu, E_{\text {des }}$ ranging from $10^{12} \mathrm{~s}^{-1}, 1.38 \mathrm{eV}$ to $10^{14}$ $\mathrm{s}^{-1}, 1.56 \mathrm{eV}$. This is in qualitative agreement with the diffraction data and supports the assumption that desorption is the main mechanism in writing the grating.

\section{Discussion}

To obtain the variation of the SH signal with incident angle (fig. 2) the use of wedged substrates was found to be essential to suppress SH signals that arrive at the detector via reflection of fundamental and/or second-harmonic at the rear of the glass substrate.

No linear diffraction from the gratings was found. For a linear experiment the modulation is extremely small since the thickness of the monolayer $\approx 1 \mathrm{~nm}$ and refractive index of $\approx 1.5$ give a phase retardation of only $0.4^{\circ}$ and a negligible amplitude attenuation. The calculated diffracted intensity is about $10^{-6}$ of the incident intensity, which is less than the randomly scattered light. On the other hand, for second-harmonic generation the $20 \%$ modulation in $\chi^{(2)}$ allowed us to detect both in transmission and reflection the diffracted orders from $n=-3$ to $n=3$.

To calculate the variation in the $\mathrm{SH}$ signal during thermal desorption (fig. 3), we used $E(2 \omega) \propto$ $\chi^{(2)} \propto N$. The good agreement with experiment confirms the validity of the linear relationship between $\chi^{(2)}$ and $N$ in this density range. This implies that the calculated modulation in $\chi^{(2)}$ (see inset fig. 2 ) directly corresponds to a modulation in adsorbate density $N$. We expect thermal desorption to be the dominant mechanism in writing the grating. Photochemical effects are not expected to play a significant role. For the given irradiation conditions and an adsorbate density $N=1 \times 10^{14} \mathrm{~cm}^{-2}$ each molecule is excited on average by only 350 photons.

Finally, calculation of the surface temperature with a one-dimensional model is justified only when the laser focus spot diameter $l$ exceeds the thermal diffusion length i.e. $l \gg(4 K t / \rho c)^{1 / 2}$. In our situation, with $l=0.7 \mu \mathrm{m}$ we calculate a diffusion length of 0.5 $\mu \mathrm{m}$. This indicates that we are just at the limit of the validity of the model.

\section{Conclusions}

We have shown that high-quality structured monolayers can be obtained by a laser ablation technique. The angular dependence of the SH diffraction signals in a reflection experiment is well described by a slight modification of existing theory. The power ratio of the diffraction orders provides information on the spatial profile structured in the monolayer. The modulation depth obtained is in good agreement with expectations based on a model for thermal desorption, with a surface temperature calculated from a heat conduction equation. Finally, these 
results may serve as a basis for experiments on (transient) gratings to study the nonlinear optical properties of surfaces.

\section{Acknowledgement}

We would like to thank Ing. J. Wijn and Dr. P. Put for production of the monolayer gratings and Drs. M. Barmentlo for his assistance during the desorption measurements.

\section{References}

[1] Y.R. Shen, Chemistry at Surfaces and Interfaces, eds. R.B. Hall and A.B. Ellis (V.C.H. Publishers Inc., Florida, 1986).

[2] G.L. Richmond, J.M. Robinson and V.L. Shannon, Progr. in Surf. Sci. Vol. 28 (1988) 1.

[3] H.W.K. Tom, T.F. Heinz and Y.R. Shen, Laser Chem. 3 (1983) 279

[4] X.D. Zhu, Th. Rasing and Y.R. Shen, Phys. Rev. Lett. 61 (1988) 2883.
[5] X.D. Zhu and Y.R. Shen, Optics Lett. 14 (1989) 503.

[6] G. Reider, M. Huemer and A.J. Schmidt, Optics Comm. 68 (1988) 149.

[7] T. Suzuki and T.F. Heinz, Optics Lett. 14 (1989) 1203.

[8] A. Bratz, B.U. Felderhof and G. Marowsky, submitted to Appl. Phys. B.

[9] H.J. Eichler, P. Gunter and D.W. Pohl, Laser induced dynamic gratings (Springer, Verlag, Berlin, 1986).

[10] G. Marowsky, L.F. Chi, D. Möbius, R. Steinhoff, Y.R. Shen, D. Dorsch and B. Rieger, Chem. Phys. Lett. 147 (1988) 420 .

[11] T.F. Heinz, H.W.K. Tom and Y.R. Shen, Phys. Rev. A 28 (1983) 1883.

[12] V. Mizrahi and J.E. Sipe, J. Opt. Soc. Am. B 5 (1988) 660.

[13] D. Menzel, Desorption phenomena, in Topics Applied Physics Vol. 4 (Springer Verlag, New York, 1975) p. 102.

[14] J.H. Bechtel, J. Appl. Phys. 46 (1975) 1585.

[15] P. Guyot-Sionnest, Y.R. Shen and T.F. Heinz, Appl. Phys. B 42 (1987) 237.

[16] B. Dick, A. Gierulsky and G. Marowsky, Appl. Phys. B 38 (1985) 107.

[17] A. Bratz and G. Marowsky, submitted to Optics Comm.

[18] C.S. Mullin, P. Guyot-Sionnest and Y.R. Shen, Phys. Rev. A. 39 (1989) 3745 . 